The LSST camera corner raft conceptual design: a front-end for guiding and wavefront sensing

K. Arndt, V. Riot, E. Alagoz, A. Biccum, A. Bohn, J. Clampit, T. Coirot, W. Cui, L. Hoffman, A. Lichti, D. Skaggs, I. Shipsey, M. Triano, B. Xin, K. Ziegler, J. Oliver, R. Van Berg, G. Haller, L. Sapozhnikov, S. Olivier

May 26, 2010

The LSST camera corner raft conceptual design: a front-end for guiding and wavefront sensing San Diego, CA, United States June 27, 2010 through July 2, 2010 
This document was prepared as an account of work sponsored by an agency of the United States government. Neither the United States government nor Lawrence Livermore National Security, LLC, nor any of their employees makes any warranty, expressed or implied, or assumes any legal liability or responsibility for the accuracy, completeness, or usefulness of any information, apparatus, product, or process disclosed, or represents that its use would not infringe privately owned rights. Reference herein to any specific commercial product, process, or service by trade name, trademark, manufacturer, or otherwise does not necessarily constitute or imply its endorsement, recommendation, or favoring by the United States government or Lawrence Livermore National Security, LLC. The views and opinions of authors expressed herein do not necessarily state or reflect those of the United States government or Lawrence Livermore National Security, LLC, and shall not be used for advertising or product endorsement purposes. 


\title{
The LSST camera corner raft conceptual design: a front-end for guiding and wavefront sensing
}

\author{
Kirk Arndt ${ }^{\mathrm{a}}$, Vincent Riot ${ }^{*}$, Enver Alagoz ${ }^{\mathrm{a}}$, Alec Biccum ${ }^{\mathrm{a}}$, Andy Bohn ${ }^{\mathrm{a}}$, Joseph Clampit ${ }^{\mathrm{a}}$, Tony \\ Coiro $^{a}$, Wei Cui ${ }^{a}$, Liz Hoffman ${ }^{a}$, Alan Lichti ${ }^{a}$, Desiree Skaggs ${ }^{a}$, Ian Shipsey ${ }^{a}$, Matt Triano ${ }^{a}$, Bo Xin ${ }^{a}$, \\ Kat Ziegler ${ }^{\mathrm{a}}$, John Oliver ${ }^{\mathrm{c}}$, Richard Van Berg ${ }^{\mathrm{d}}$, Gunther Haller ${ }^{\mathrm{e}}$, Leonid Sapozhnikov ${ }^{\mathrm{e}}$, Scot Olivier ${ }^{\mathrm{b}}$ \\ ${ }^{a}$ Purdue University, West Lafayette, IN, USA 47907-1285; \\ ${ }^{\mathrm{b}}$ Lawrence Livermore National Laboratory, 7000 East Ave., Livermore, CA USA 94550 \\ ${ }^{\mathrm{c}}$ Harvard University, Cambridge, MA USA 02138 \\ ${ }^{\mathrm{d}}$ University of Pennsylvania, Philadelphia, PA USA 19104-6396 \\ ${ }^{\mathrm{e}}$ Stanford Linear Accelerator Center, Stanford, CA 94309
}

\begin{abstract}
The Large Synoptic Survey Telescope (LSST) is a proposed ground based telescope that will perform a comprehensive astronomical survey by imaging the entire visible sky in a continuous series of short exposures. Four special purpose rafts, mounted at the corners of the LSST science camera, contain wavefront sensors and guide sensors. Wavefront measurements are accomplished using curvature sensing, in which the spatial intensity distribution of stars is measured at equal distances on either side of focus by CCD detectors. The four Corner Rafts also each hold two guide sensors. The guide sensors monitor the locations of bright stars to provide feedback that controls and maintains the tracking of the telescope during an exposure. The baseline sensor for the guider is a Hybrid Visible Silicon hybrid-CMOS detector. We present here a conceptual mechanical and electrical design for the LSST Corner Rafts that meets the requirements imposed by the camera structure, and the precision of both the wavefront reconstruction and the tracking. We find that a single design can accommodate two guide sensors and one split-plane wavefront sensor integrated into the four corner locations in the camera.
\end{abstract}

Keywords: LSST, guide sensors, wavefront sensors, ground-based telescope

\section{INTRODUCTION}

The LSST camera will be the largest digital camera ever constructed. The camera assembly is 1.6 meters by 3 meters and weighs $\sim 2800$ kilograms (see Figure 1 left). It is a large-aperture, widefield optical imager designed to provide a $3.5^{\circ}$ field of view with better than 0.2 arcsecond resolution. The image focal plane is flat with a diameter of $\sim 64 \mathrm{~cm}$. The camera includes a filter changing mechanism and shutter. The camera will produce extremely high quality data with minimal downtime and maintenance. The focal plane operates at a temperature of $-100^{\circ} \mathrm{C}$ to achieve desired detector performance. The focal plane is contained within an evacuated cryostat[1], which incorporates detector electronics and thermal control. The last of three LSST lenses, L3, serves as the entrance window for the focal plane and as part of the vacuum seal of the cryostat.

The 3.2 giga-pixel focal plane array is comprised of 189 CCD sensors with 10 micron pixels. The sensors are deepdepletion, back-illuminated devices with a highly segmented architecture that enables the entire array to be read out in 2 seconds. The detectors are grouped in identical $3 \times 3$ arrays called "Science Rafts". Each science raft includes dedicated front-end electronics boards in a "Tower" that fits within the footprint of its sensors, thus forming a 144-megapixel camera on its own (see Figure 1 right). The science rafts are mounted on a silicon carbide "Grid" inside the cryostat and associated electronics are mounted on the cryo-plate. The grid also contains four sets of guide sensors and wavefront sensors in "Corner Rafts" at the edge of the camera field. 

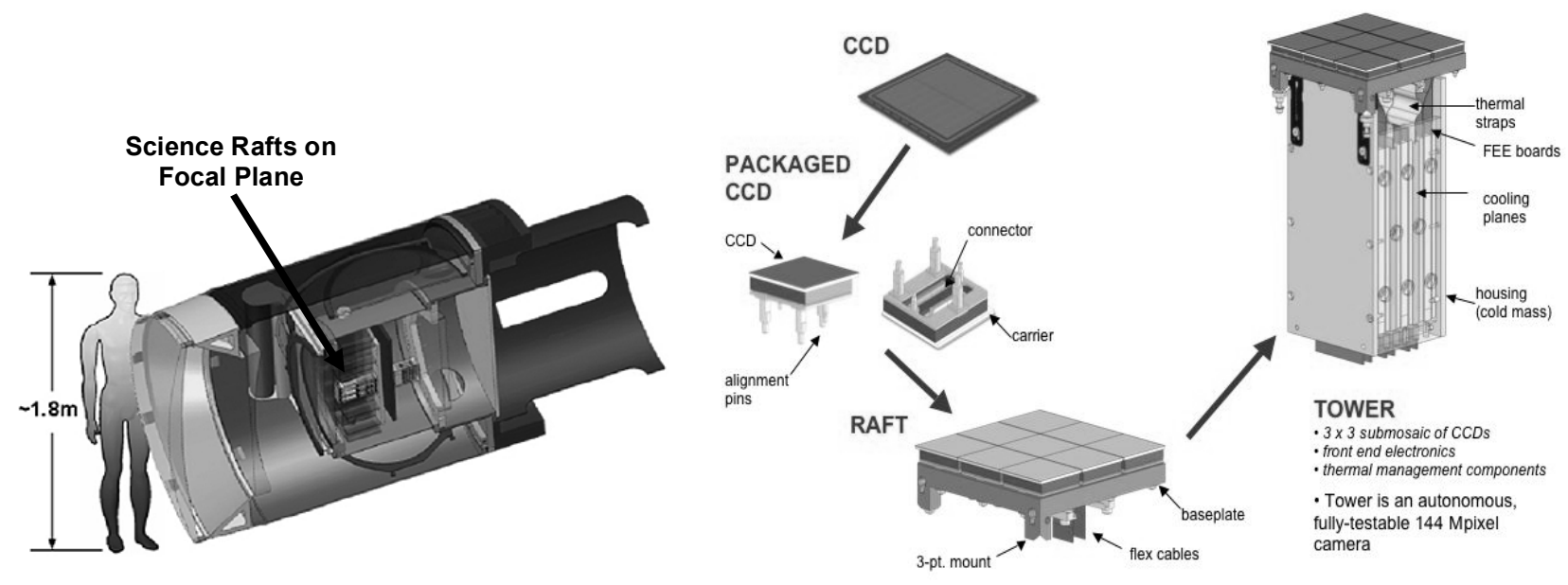

Figure 1. (Left) Cut-away view of LSST Camera assembly (Right) LSST sensor-to-science raft/tower configuration

\section{CORNER RAFT FUNCTIONALITY}

\subsection{Role of the corner raft in the LSST}

The corner raft sub-system is contained inside the LSST Camera as it needs access to the focal plane, but has a role in activities encompassing the entire operation of the telescope.

The corner raft sub-system is responsible for acquiring pixel data for the active optics system based on curvature wavefront sensing [2][3]. To support this activity, the corner raft must acquire intra and extra focal images at 4 locations at the edges of the focal plane that will be used by the Telescope Control System to estimate the wavefront and, through reconstruction, provide inputs to the active optics controller. Images are collected in parallel with the science images and follow the corresponding exposure time and cadence (nominally 15 second exposure and 2 second readout time).

The corner raft sub-system is also responsible for acquiring small images centered on selected bright stars at a nominal $9 \mathrm{~Hz}$ rate. These images are used to estimate the pointing variation during the exposure and provide input to the telescope control tracking system.

\subsection{Corner rafts as LSST camera sub-systems}

The corner raft sub-system will provide images from 8 guide sensors and 4 wavefront split sensors. The images are collected at the focal plane by the sensors. The sensors are readout by the analog electronics located in the corner raft towers. As shown in Figure 2, the front end electronics in each corner raft tower, identified by Wavefront FEB1, Wavefront FEB2, Guider FEE1 and Guider FEE2, reads the data from the sensors and provides a conditioned signal to the back end electronics located in the corner raft control crates. The back end electronics provide an interface to the camera control and data systems over a high speed serial interface (PGP link). The Timing and Control Module (TCM) provides real time synchronization across the camera to ensure that data is acquired with accurate rate, start and stop times. The Wavefront Data System (WDS) provides access to the wavefront images to either the Telescope Control System (TCS) for close loop operation or to the data management system for archival storage. The Guider Data System (GDS) computes in real time the centroid location of the selected guide stars and provides access to the data from the TCS for tracking. The telescope decides whether the information is used to close the loop or not, depending on the current mode of operation. The Observatory Control System (OCS) initiates commands resulting in images being acquired by the corner raft sub-system[4]. The OCS coordinates with the Camera Control System (CCS) for camera specific actions including the corner raft operating steps and the TCS for data processing and active control. 




Figure 2. Corner raft system component block diagram in the context of the LSST camera and telescope

\section{CORNER RAFT SENSORS AND PACKAGING}

\subsection{Guide sensors and package}

Two guide sensors are located in each corner raft (total of 8 guide sensors - see Figure 3). The baseline design for guiders uses commercially available $2 \mathrm{~K} \times 2 \mathrm{~K}$ HyViSi/H2RG[5] Teledyne (formerly Rockwell Scientific) pin-CMOS hybrid detector assemblies with 18um pixels, packaged for ground-based astronomy. The H2RG multiplexer provides a straightforward means of reading out region-of-interest (ROI) windows from the pin-CMOS sensor due to the amplifierper-pixel architecture and flexible addressing modes. Pixels can be reset at any time providing the electronic shuttering. The HyViSi/H2RG assembly is supported by a sophisticated control and data acquisition ASIC "SIDECAR" [5], also available commercially from Teledyne, offering compactness, cryogenic operation, low power, and full digital output. Each SIDECAR ASIC is mounted on a custom printed circuit board and housed inside a metal enclosure for EMI shielding and heat transfer to the tower. A flat flex cable mates to a pin grid array in the multi-layer ceramic circuit on 
the back-side of the guide sensor package, and terminates in an 85-pin Nano-connector for connection to the SIDECAR ASIC board.

\section{LSST FOCAL PLANE}

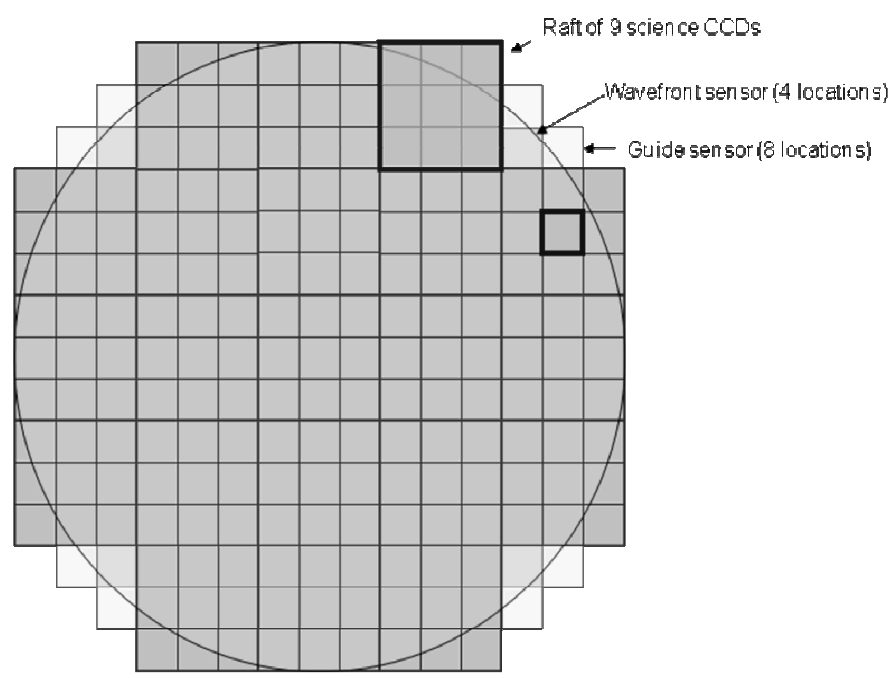

Figure 3. LSST focal plane layout

\subsection{Wavefront sensors and package}

LSST requires four wavefront sensors located in the corner rafts at the periphery of the focal plane (see Figure 3). A depiction of the wavefront sensor concept is shown in Figure 4. Two $2 \mathrm{~K}$ x $4 \mathrm{~K}$ segmented CCDs will be packaged with an offset relative to the science focal plane to produce intra and extra focal images. An offset of roughly $+/-1 \mathrm{~mm}$ from the science focal plane is anticipated. Wavefront sensor CCDs will share the layout and processing of the science sensors, and have similar performance as science CCDs regarding quantum efficiency, point spread function, read noise, and readout speed. Detailed mechanical specifications such as fill factor, flatness, parallelism of the intra and extra focal sections, and temperature sensing will be determined by results of the validation of this wavefront sensing scheme by laboratory measurements.



Focal Plane

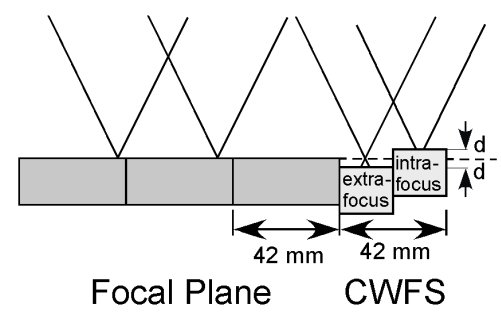

Focal Plane

Figure 4. Wavefront sensor system split detector

\subsection{Sensor Corner raft}

Each sensor package will be mounted to the corner raft structure by means of 3 threaded stud/pin legs, Belleville washers and locking nuts. Removable, thermally-conductive spacer/shims that can be adjusted will be used to set the height of the sensor surfaces above the corner raft. Identical corner rafts will be mounted in four corner locations on the grid structure (which also supports the science rafts) by means of an adjustable 3-point ball-and-vee kinematic mount design[6]. A concept for this mount system is shown in Figure 5. 


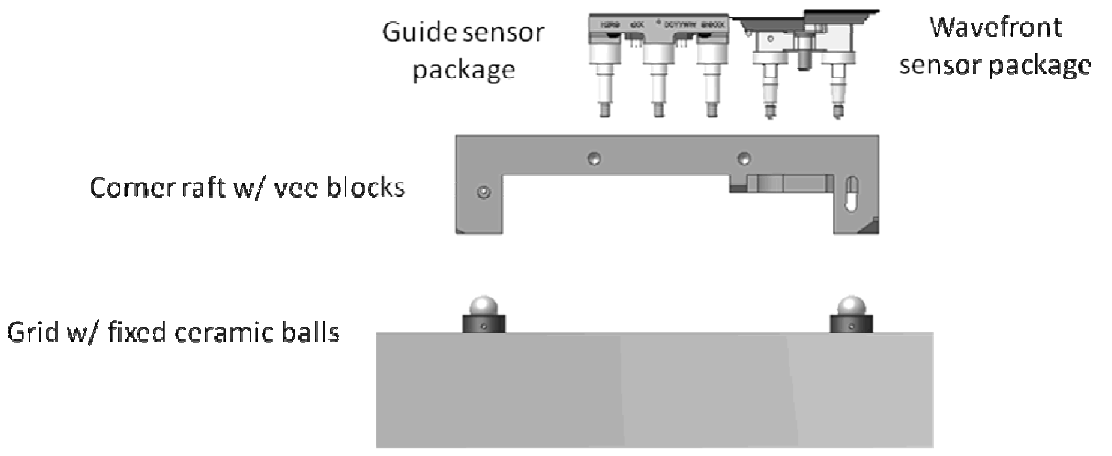

Figure 5. Side view of sensor-to-corner raft-to-grid mounting scheme

The back side of the corner raft features three radially-arrayed vees ground into the raft material. The vees uniquely define the position of the corner raft with respect to silicon-nitride ceramic balls that are fixed in cups mounted on the grid. This forms a kinematic connection that isolates the corner (and science) rafts from grid distortion due to external dynamic and transient loads, assembly tolerances, and to the expected thermal motions due to differential contraction during cooling. The corner rafts are held in position by springs that pre-load the corner rafts against their kinematic coupling to the grid, producing a uniform, invariant loading. The deflection of grid and corner raft due to the spring loading is compensated for during initial integration, and remains unchanged in operation, independent of camera orientation and temperature.

The grid, science rafts, and corner rafts will be manufactured from a silicon-carbide ceramic matrix composite. This material is used for lightweight and stable space-borne structures, including focal planes, optical benches, and mirrors. The high modulus, high thermal conductivity, near-zero expansion coefficient and improved fracture toughness of the ECM CeSic ${ }^{\circledR}$ material makes it ideal for the grid, science raft, and corner raft structures. A closed-loop thermal control system will be used to adjust the temperature of the corner rafts, thereby maintaining a suitable and stable operating temperature for the sensors.

\section{CORNER RAFT MECHANICAL AND ELECTRICAL ASSEMBLY}

\subsection{Corner raft tower concept}

The LSST Camera cryostat contains four sets of guide sensors and wavefront sensors in corner rafts at the edge of the camera field (see Figure 6 left). Each Corner Raft/Tower contains one wavefront sensor and two guide sensors and dedicated front end electronics. The mechanical and thermal design of the corner rafts is as similar as possible to the science rafts.

Front-end electronics for operating the wavefront and guide sensors are packaged within the volume behind the detectors, similar to the science raft configuration, in an electronics crate tower. The grid supports as little mass as possible - only the sensor rafts and their supports - to reduce gravity induced deflections. The tower is supported by a neighboring cryogenically-cooled "Cryoplate" at about -130C temperature. Each corner raft is thermally connected, but structurally decoupled from the tower by use of thin copper thermal straps to remove the electrical and radiant heat load from each Corner Raft and conduct it back to the cryoplate. Electrical connections between sensors and front-end electronics boards are made by flat flex circuit cables. Details of the preliminary design for corner raft/towers for the LSST camera are shown in Figure 6 (right). 

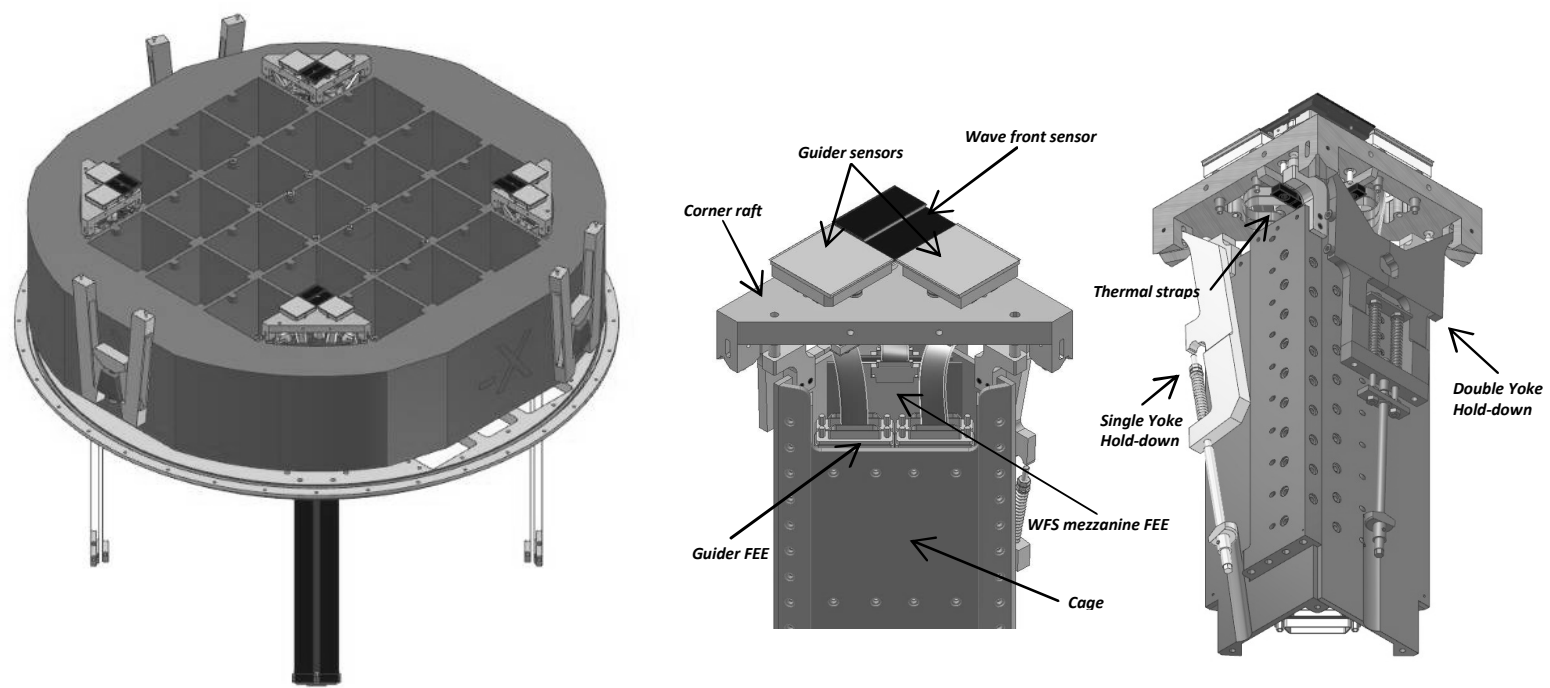

Figure 6. (Left) Corner raft/towers with FE-BE cables on grid and cryoplate (Right) Corner raft/tower

Data acquisition and control for the wavefront and guide sensors are managed using the same infrastructure as for the science detectors. The corner raft tower contains two SIDECAR ASIC for reading the guide sensor data and two ASPIC ASIC for CCD readout[7] developed specifically for LSST (see Figure 7). The wavefront electronics used to read the CCD sensors are identical to the science electronics and are simply re-packaged to accommodate the smaller footprint and reduced numbers of channels.
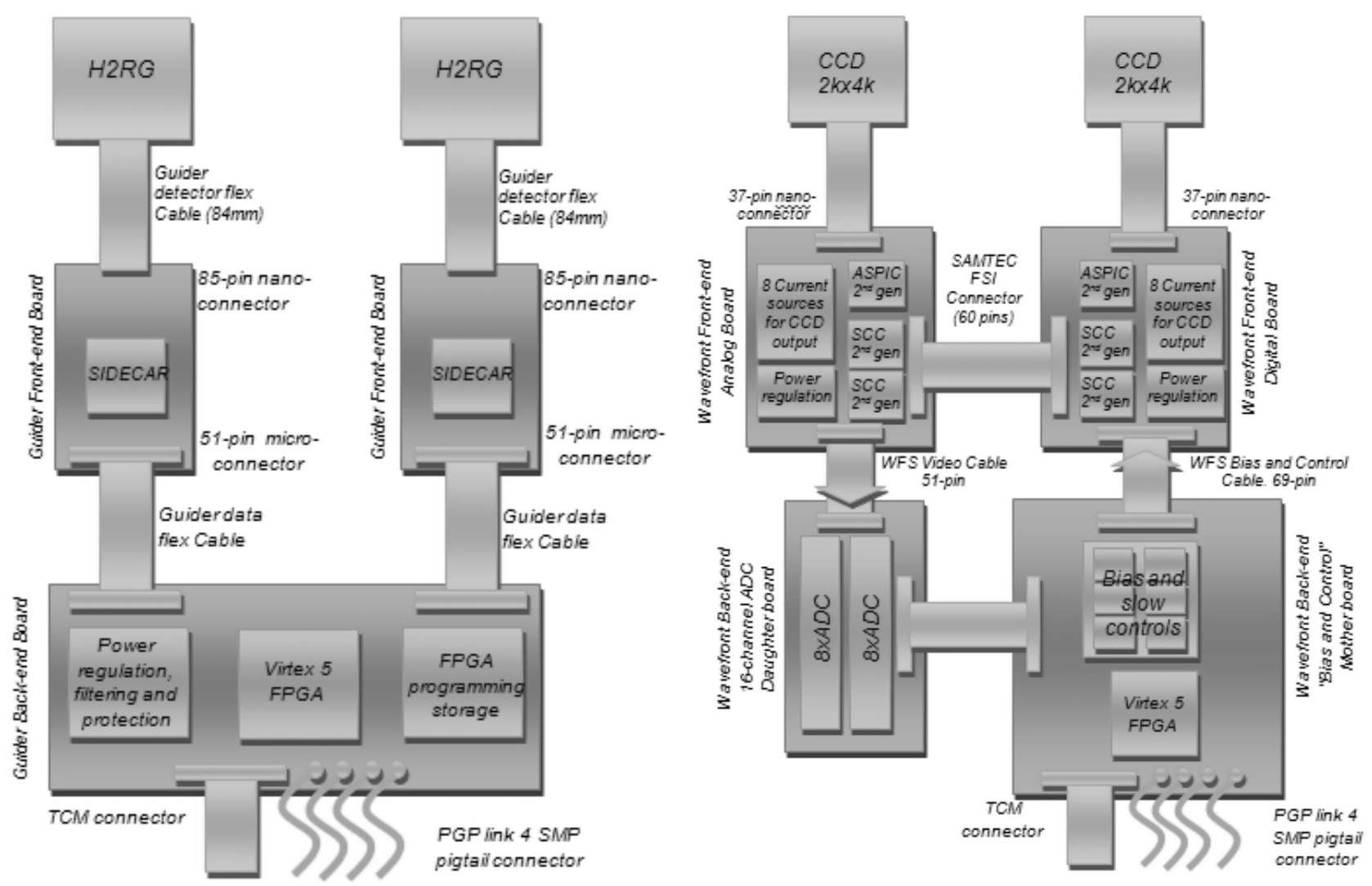

Figure 7. (Left) Corner raft Guider electronics (Right) Corner raft Wavefront electronics 


\subsection{Corner raft control crate concept}

Back end electronics for operating the wavefront and guide sensors are packaged within the volume behind the corner raft/towers in "Control Crates" (see Figure 8 left). The control crates are supported by a neighboring cryogenic-cooled "Cold Plate" at about $-40 \mathrm{C}$ temperature. The cold plate removes process heat from the power, clock, and digital back end electronics. Flat flex cables are used to make the electrical connections between front end electronics boards in the tower and back end electronics boards in the control crates (see Figure 8 right).
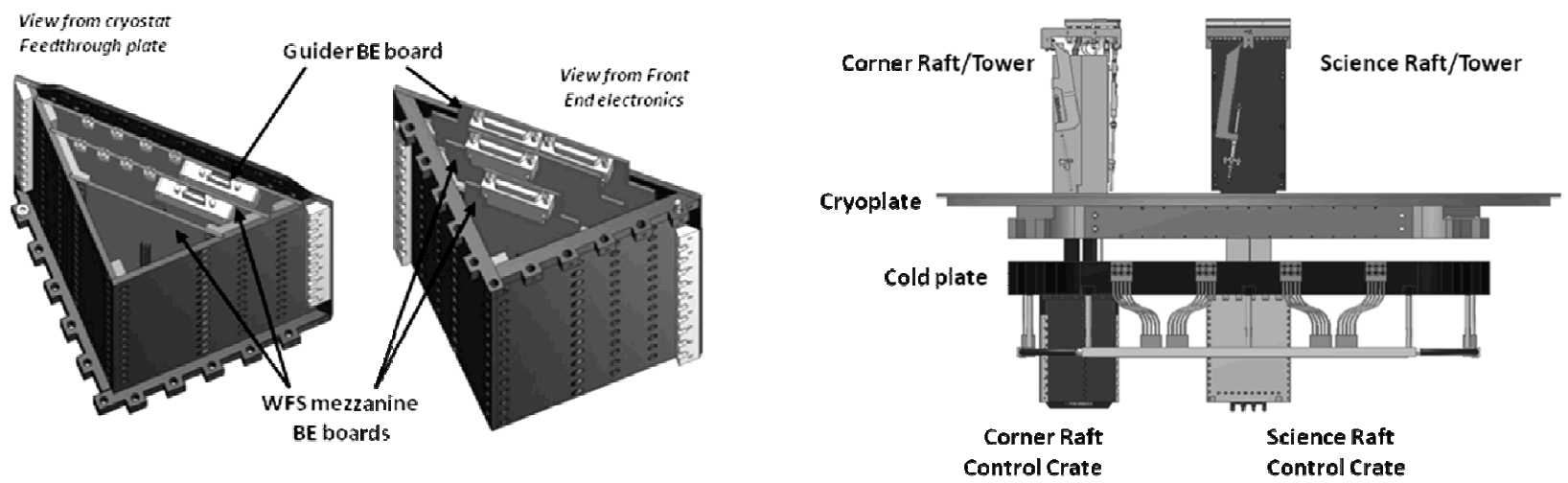

Figure 8. (Left) Corner raft control crate preliminary design (Right) Raft/tower to control crate stack

\subsection{Thermal and structural analysis}

Thermal steady-state finite element analysis results of a simplified corner raft/tower model, with average power heat load and temperature in the cryoplate cooling channels fixed at $-130 \mathrm{C}$, are shown in Figure 9. Makeup heat of $\sim 1.2 \mathrm{~W}$ (from two ohmic heaters mounted on the backside of the corner raft) is required to raise the temperature of the sensors to -100C. A closed-loop thermal circuit will be used to adjust the make-up heat on each corner raft to maintain a stable operating temperature for the sensors with varying thermal loads.

Preliminary structural finite element analysis of a simplified corner raft and sensors model, with the corner raft supported by the vees on fixed balls, and including thermal loads, gravity and applied spring forces, results in a " $\mathrm{z}$-axis" deformation of the corner raft and sensor surfaces of less than 1 micron.

Future engineering studies will include thermal and structural FEA of detailed models and transient loads.
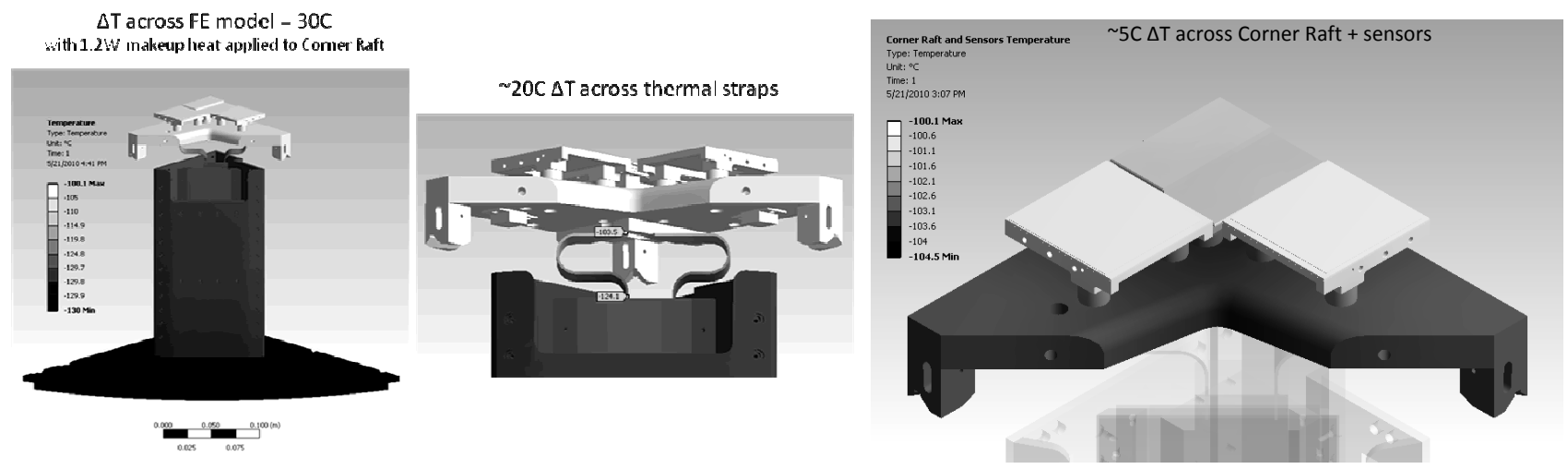

Figure 9. (Left) Corner raft/tower thermal analysis results (Right) Steady state thermal gradient of a corner raft with sensors 


\subsection{Assembly and testing plans}

\subsubsection{Preliminary assembly concept}

Purdue University is responsible for assembling and testing the corner raft/towers. Given that LSST is a survey telescope, the mechanical and electrical systems must be reliable, with a high up-time. All mechanical and electrical subassemblies will be functionally and performance tested prior to installing in the corner raft/towers, minimizing the risk of early failures and the need for disassembly during subsequent camera integration and commissioning. Sensors and electronic sub-assemblies are modular and self-contained, allowing them to be installed, removed, and serviced with minimal disturbance of neighboring components.

Sensors will likely be assembled into corner rafts in a face-down orientation to allow easy access to the installation mechanism and to minimize the potential for surface contamination and accidents. Special tapered installation pins are screwed onto the ends of two of the threaded stud legs on the sensor package. Using a handling fixture, the sensor package (with flex cable attached) is pulled into contact with the corner raft. The taper on the pins is designed to fully engage the mounting holes in the corner raft before the sensor package overlaps any neighboring sensors.

After sensors are installed and leveled in the corner raft, a protective lid will be fastened to the corner raft to protect the sensors from particulates and contamination. Subassemblies (consisting of wavefront sensor front end electronic boards fastened to mounting bars, SIDECAR electronics boards in enclosures and fastened to one tower cage side, cage sides joined to form the other two sides of the tower) will be pre-assembled before final integration. The thermal straps, front end electronics and spring-loaded yoke hold-down sub-assemblies are progressively connected to the corner raft, sensor flex cables, and tower, working from the center to the outside of the corner raft and tower. A conductance barrier (to separate the ultra-clean focal plane from the support electronics) is formed during assembly by the installation of barshaped parts between thermal straps and sensor flex cables across the front end of the tower. The assembly of the back end electronics boards to mounting bars, and subsequent assembly into the control crates proceeds similarly to the assembly of front end boards in the tower.

After assembly and system testing, each corner raft/tower and control crate will be shipped to SLAC for integration into the LSST Camera. The same procedure and installation tooling on a gantry motion control system envisioned for integration of science raft/towers and control crates will be used for integration of corner raft/towers and control crates into the camera cryostat.

\subsubsection{Validation of the simulation of the wavefront sensing by laboratory measurements}

Accurate wavefront sensing is critical for LSST to reach its science goals, but the LSST wavefront sensing method has not been used before. Simulations performed at LLNL predict the method will work, but no test data exists. To develop confidence for the LSST wavefront sensing method, an optical wavefront testbed has been built (depicted in Figure 10 left). It has a capability of producing aberrated images that can be used to develop wavefront reconstruction algorithms or to improve existing ones. Low order aberrations such as astigmatism, coma are introduced into a collimated beam by means of different lens configurations. A special focusing lens ( $\mathrm{f} / 1.23)$ provides an LSST-like fast beam. A CCD camera with pixel size (9 microns) similar to the LSST science sensors (10 microns) records the images. Moving the camera in and out of focus provides defocused images.
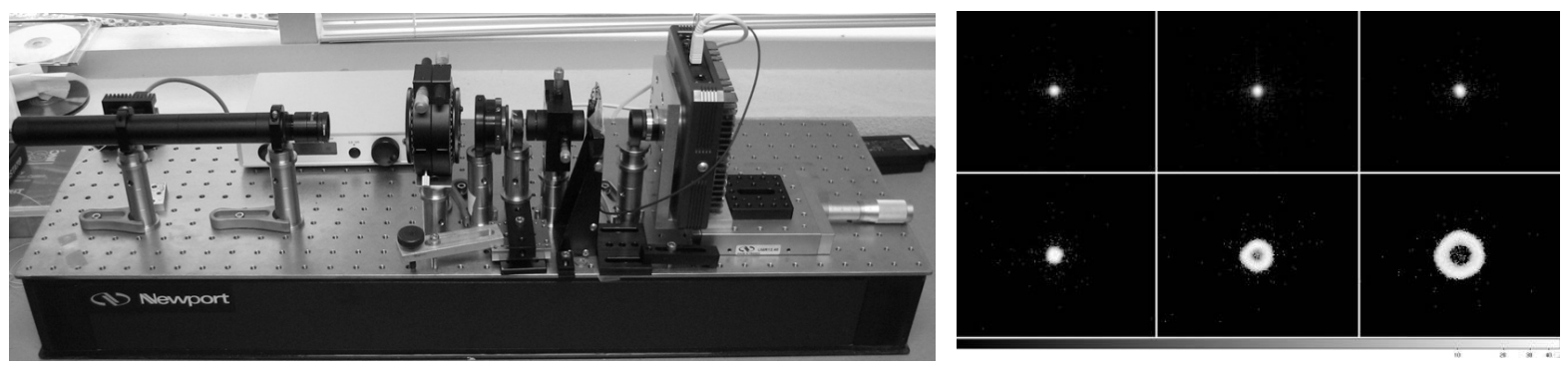

Figure 10. (Left) Optical wavefront testbed at Purdue University. (Right) Simulated images of star produced when the camera is in the focal plane, or displaced from the focal plane. Displacements shown are: Top row: 0, 15, 50 microns Bottom row: 100, 300, 500 microns. In LSST, wavefront sensors will be displaced by $+/-1000$ microns from the focal plane. 
An end-to-end photon simulation of LSST wavefront sensing is shown in Figure 10 (right). Recording images from simulation on each side of focus enables reconstruction of wavefront aberrations caused by atmospheric turbulence, mirror aberrations and other sources of noise.

In addition to the optical wavefront testbed, an LSST beam simulator is under construction at UC Davis and could be used for testing curvature wavefront sensors and software. The LSST beam simulator is designed to simulate the fast beam of the LSST telescope, including the central obscuration, and to provide nearly diffraction limited images over an entire 4K x 4K CCD sensor area. The wide field of the beam simulator will enable lab tests under a variety of conditions such as vignetting, wavefront sensor tilt, star crowding, stellar density, and $\mathrm{S} / \mathrm{N}$ ratio.

\subsubsection{Testing corner raft sensors}

All Corner Raft sensor tests are to ensure that both wavefront and guider sensors meet the LSST corner raft sensor requirements under LSST standard operating conditions.

The test station apparatus includes a cryostat Dewar (liquid nitrogen cooling + vacuum system), CCD and CMOS controllers, x-ray source (for the accurate sensor gain measurements), light source, monochromator, focusing optics (+ motion control), filters and mechanical shutter, integrating sphere, photosensitive diodes, a residual gas analyzer and instrumentation. All instruments are mounted on a research grade optical table that provides stiffness and vibration isolation. The sensor test station at Purdue University is initially configured for tests using a single LSST sensor that has already been thoroughly tested and understood by the SLAC/BNL team. Following single sensor characterization, the cryostat will accommodate a full corner raft/tower to be tested at cryogenic temperature.

The Purdue test station schematic layout is shown in Figure 11. Imaging detectors are mounted on a support or corner raft inside the cryostat, close to the window. Monochromatic light is sent either to the integrating sphere or to a spot projector. The integrating sphere produces flat field light that illuminates the detectors. A set of identical photosensitive diodes provides the light intensity calibration. All test station instruments are controlled from a PC via GPIB or RS232/USB connections. National Instruments LabVIEW software is currently used to control test station instruments, and detector specific software is used to control CCD/CMOS sensors. RTS2 software[8] for automated control of test station instruments and integration with image acquisition and processing is under consideration as an upgrade to the test station.

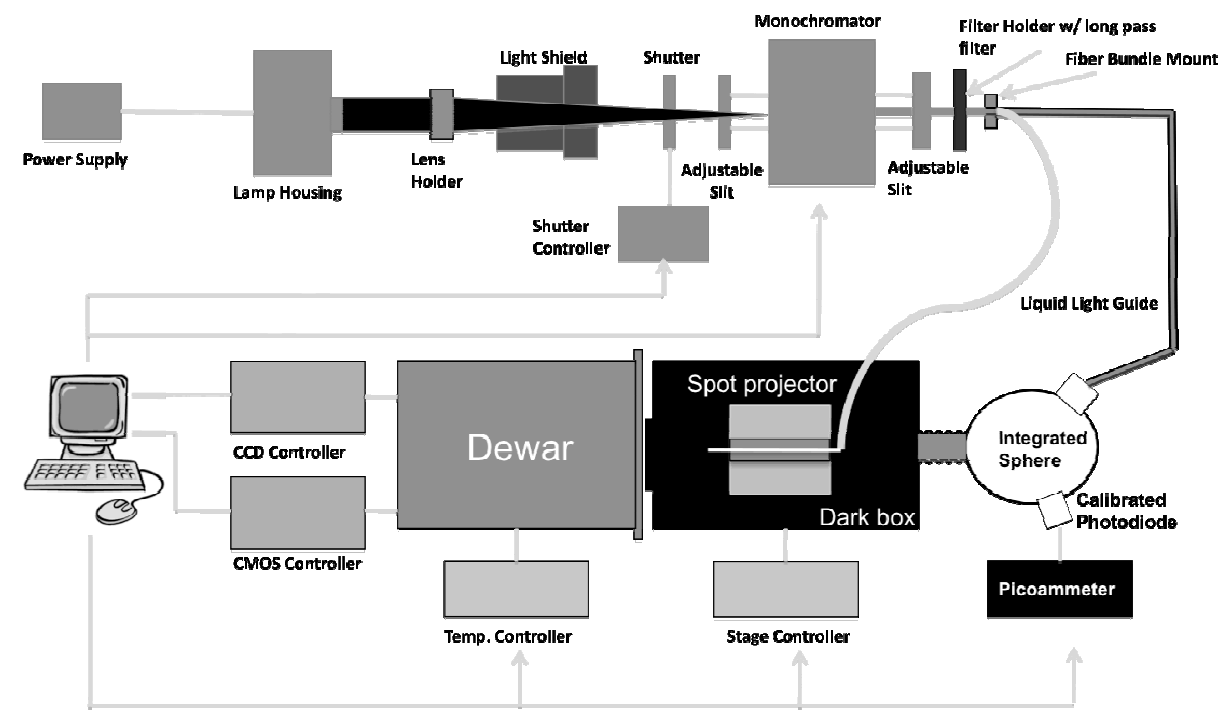

R5-232 / USB or GPIB

Figure 11. Sensor test station at Purdue University 
Upon completion of the initial CCD sensor characterization measurements, the test station capabilities will be expanded to test CMOS guider sensors in close collaboration with SLAC and RIT. Full corner raft assembly system tests will be performed following individual sensor testing. The system tests include operational tests using full corner raft electronics, sensor functionality tests and thermal performance measurement.

A metrology setup will be built to measure corner raft sensor package flatness and tip/tilt stability. The setup will consist of a laser displacement sensor on 3-axis motion stages mounted to the cryostat front cover to survey individual sensors, or sensors mounted on a corner raft, at cryogenic temperature.

Corner raft/tower testing will be performed in a future assembly and test facility at Purdue University (see Figure 12). The planned facility is a dedicated 650 square feet laboratory with provision for:

- Lab access control

- Dark room

- Electrostatic discharge controls

- Two test stations (support for concurrent assembly and testing of single sensors or corner raft/towers)

- Interior soft wall clean room (class 10,000 or better) for one or both stations

- Dedicated clean area (class 100) within clean room for unpacking sensors, assembling corner rafts, integrating in cryostat.

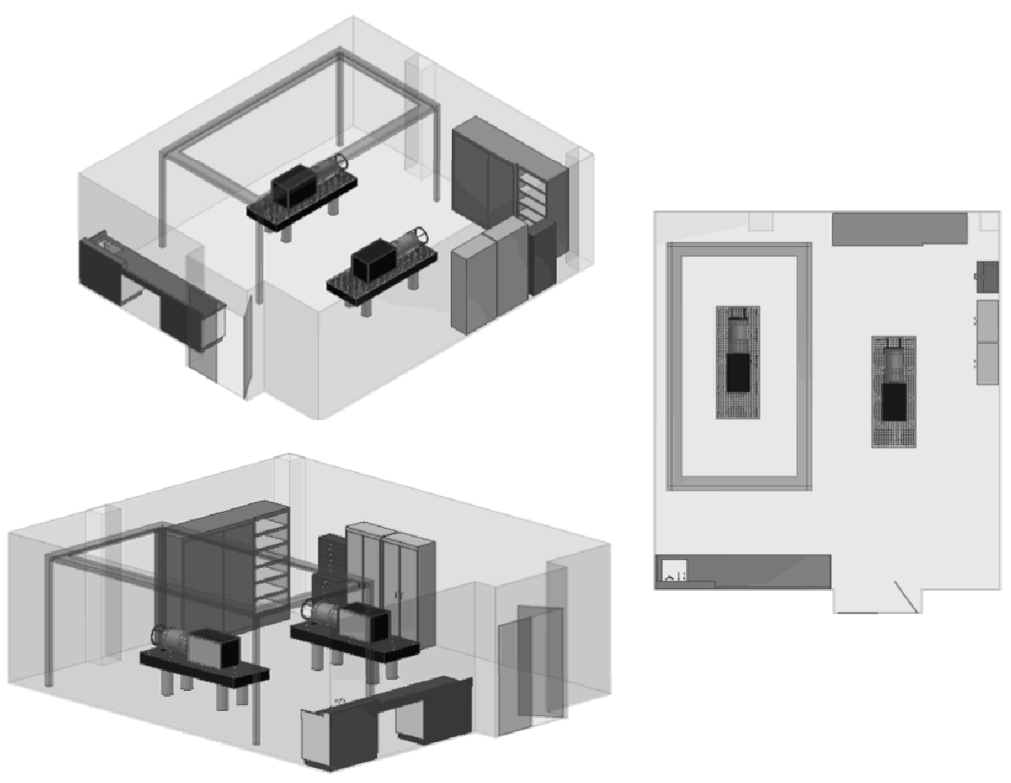

Figure 12. Future LSST corner raft assembly and test facility at Purdue University.

\section{CONCLUSION}

The mechanical and electrical conceptual design described here for the corner raft, front end tower and back end control crate is compatible with the physical constraints imposed by the LSST camera structure, and meets the performance requirements for both wavefront reconstruction and tracking. A single conceptual design accommodates two guide sensors and one split-plane wavefront sensor and can be integrated into the four corner locations at the periphery of the focal plane. The mechanical, thermal and electrical design of the corner raft subsystem is as similar as possible to the science raft subsystem, benefitting from modular and self-contained sub-assemblies that aid system verification and serviceability. Likewise, the data acquisition and control for corner raft sensors is managed using the same infrastructure as for the science detectors in the LSST camera. 


\section{REFERENCES}

[1] M. Nordby, G. Bowden, M. Foss, G. Guiffre, J. Ku, and R. Schindler, "Mechanical Design of the LSST Camera," Proc. SPIE 7018, 70182H (2008)

[2] Anastacia M. Manuel, Donald W. Phillion, Scot S. Olivier, Kevin L. Baker, and Brice Cannon, "Curvature wavefront sensing performance evaluation for active correction of the Large Synoptic Survey Telescope (LSST)," Opt. Express 18, 1528-1552 (2010)

[3] D. W. Phillion, S. S. Olivier, K. Baker, L. Seppala, and S. Hvisc, "Tomographic wavefront correction for the LSST," Proc. SPIE 6272, 627213 (2006)

[4] Stuart Marshall, Jon Thaler, Terry Schalk, and Michael Huffer, "LSST camera control system," Proc. SPIE 6274, $627422(2006)$

[5] J.W. Beletic, R. Blank, D. Gulbransen, D. Lee, M. Loose, E.C. Piquette, T. Sprafke, W.E. Tennant, M. Zandian, and J. Zino, "Teledyne Imaging Sensors: Infrared imaging technologies for Astronomy \& Civil Space", Proc. SPIE 7201, 70210H (2008)

[6] Peter Z. Takacs, Paul O'Connor, Veljko Radeka, George Mahler, James Frank, and John Geary, "LSST detector module and raft assembly metrology concepts", Proc. SPIE 6273, 62733Q (2006)

[7] R. Sefri, V. Tocut, H. Lebbolo, S. Bailey, D. Martin, P. Antilogus, C. Delataille, J. Jeglot, M. Moniez, F. Wicek, "ASPIC : LSST Camera Readout Chip", Nuclear Science Symposium and Medical Imaging Conference (2009)

[8] P. Kubánek, et al., "RTS2: a powerful robotic observatory manager," Proc. SPIE 6274, 62741V (2006)

\section{ACKNOWLEDGMENTS}

LSST is a public-private partnership. Funding for design and development activity comes from the National Science Foundation, private donations, grants to universities, and in-kind support at Department of Energy laboratories and other LSSTC Institutional Members. This work is supported by in part the National Science Foundation under Scientific Program Order No. 9 (AST-0551161) and Scientific Program Order No. 1 (AST-0244680) through Cooperative Agreement AST-0132798. Portions of this work are supported by the U.S. Department of Energy under contract DEAC02-76SF00515 with the Stanford Linear Accelerator Center, contract DE-AC02-98CH10886 with Brookhaven National Laboratory, with the Lawrence Livermore National Laboratory under the auspices of the U.S. Department of Energy under Contract DE-AC52-07NA27344 and in part by the U.S. Department of Energy Office of High Energy Physics under grant DE-FG02-91ER40681A29 awarded to Purdue University. Portion of this work was also supported in part by Purdue University.

Additional funding comes from private donations, grants to universities, and in-kind support at Department of Energy laboratories and other LSSTC Institutional Members.

This document was prepared as an account of work sponsored by an agency of the United States government. Neither the United States government nor Lawrence Livermore National Security, LLC, nor any of their employees makes any warranty, expressed or implied, or assumes any legal liability or responsibility for the accuracy, completeness, or usefulness of any information, apparatus, product, or process disclosed, or represents that its use would not infringe privately owned rights. Reference herein to any specific commercial product, process, or service by trade name, trademark, manufacturer, or otherwise does not necessarily constitute or imply its endorsement, recommendation, or favoring by the United States government or Lawrence Livermore National Security, LLC. The views and opinions of authors expressed herein do not necessarily state or reflect those of the United States government or Lawrence Livermore National Security, LLC, and shall not be used for advertising or product endorsement purposes. LLNLPROC-433560 